\title{
Conventional and microwave irradiated syntheses of new symmetric double Schiff bases of 1,1'-bis(4-amino phenyl)cyclohexane, their spectral characterization and microbial activity
}

\author{
Bhavesh J. Gangani ${ }^{1}$, Parsotam H. Parsania ${ }^{2^{*}}$ \\ ${ }^{1}$ Shree M. P. Shah Arts \& Science College, Surendranagar-363001, Gujarat, India \\ ${ }^{2}$ Department of Chemistry, Saurashtra University, Rajkot-360 005, Gujarat, India \\ ${ }^{1,2}$ E-mail id: bj2gangani@gmail.com, phparsania22@gmail.com,
}

\begin{abstract}
Keywords: Microwave irradiated syntheses, Symmetric double Schiff bases, UV, FTIR, ${ }^{1} H$ NMR, MS, bacterial activity
\end{abstract}

\begin{abstract}
Symmetric double Schiff bases of 1,1'- bis(4-amino phenyl)cyclohexane and substituted aromatic benzaldehydes were synthesized by thermal and microwave irradiated green chemistry techniques. The purity of Schiff bases was checked by TLC. The structure of Schiff bases were supported by UV, FTIR, ${ }^{1} \mathrm{HNMR}$ and MS techniques. The antibacterial and antifungal activities of Schiff bases were checked against gram positive and gram negative microbes. Schiff bases showed moderate antibacterial activity but they showed good comparable antifungal activity against chosen microbes and standard drugs.
\end{abstract}

\section{INTRODUCTION}

Schiff bases are widely used as analytical reagents, fine chemicals, medical substrates and ligands for metal complexes [1, 2] having industrial importance as antifungal and biological applications [3-6]. The Schiff bases derived from aromatic diamines and salicyaldehyde or bis salicyaldehyde possess unique characteristics of improving both antiwear and corrosion inhibition of synthetic lubricants. Schiff bases are added in lubricants and lubricating oil greases up to $5 \%$ of total composition [7]. They are also useful as a starting materials for the synthesis of drugs like antibiotics, antiallergic, antiphlogistic and antitumor [8-10]. Kuzmin et. al [11] have reported anticancer activity of a set of macrocyclic Schiff bases based on 2,6-bis(2 and 4 formyl arylosy methyl) pyridines. They have derived correlation equation between the anticancer activity and structural parameters of the molecules studied. Lozitsky et. al [12] have reported 4D-QSAR study and anticancer activity of Schiff bases synthesized by the condensation of various aliphatic and aromatic diamines with 2,6-bis(2 and 4 formyl arylosy methyl) pyridine derivatives. The results of biological activities showed that Schiff bases are active towards cell growth of the nine cell culture of human malignant tumors like leukemia, CNS cancer, colon cancer, ovarian cancer and renal cancer. They claimed that pyridine ring has a negative influence on anticancer activity, while the presence of the $\mathrm{C}=\mathrm{N}$ group with different substitutions promotes anticancer activity.

Most widely Schiff bases are synthesized by classical method catalyzed by acid at reflux temperature. Recently stoichiometric reaction of aldehyde and diamine in a suitable solvent is employed successfully for the synthesis of Schiff bases [13]. In conventional method solvent removal is a problem especially when dipolar high boiling solvents are used or isolation of product through liquid-liquid extraction. The solvent free syntheses in a microwave oven reduce the risk of hazardous explosion [14]. Solvent free microwave irradiated synthesis [15, 16] has become popular over usual homogeneous and heterogeneous reaction [17-19] due to environment friendly, economic and rapid reaction rate [20-25]. This technique provides pure product in quantitative yields and structural parameters of the molecules studied. Zheng et.al [26] have reported two novel porphyrinogen like macrocyclic Schiff base ligands by different internal cavities and characterized by elemental analysis, ${ }^{1} \mathrm{H}$ NMR, FAB-MS and IR methods. They have synthesized Schiff base ligands by thermal method. 
In present work we have reported conventional and microwave irradiated synthesis of new symmetric double Schiff bases, their spectral and microbial study.

\section{MATERIALS AND METHODS}

Solvents and chemicals used in the present investigation were of LR grade and used either as received or purified by fractional distillation. 1, 1' Bis (4-aminophenyl) cyclohexane [Scheme-1] was synthesized and recrystallized according to reported work [27, 28]. Symmetric double Schiff base [Scheme-2] were synthesized by conventional and microwave irradiated techniques [29] and crystallized from appropriated solvent system as shown in Table 1. Analytical and physical data of Schiff bases (SDSB-1 to SDSB-4) are presented in Table 2.

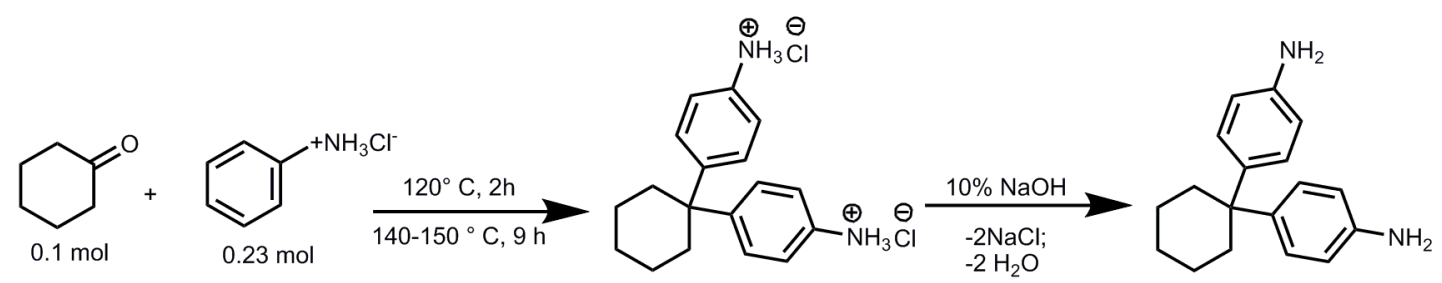

Scheme 1: Syntheses of 1,1'- bis (4-amino phenyl)cyclohexane

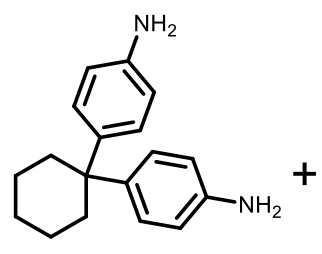

$0.01 \mathrm{~mol}$<smiles>O=Cc1ccccc1</smiles>

0.021

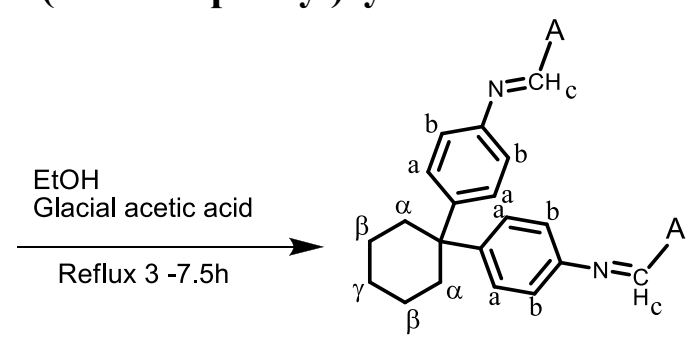

SDSB-1 to SDSB-4<smiles>CC=Cc1ccccc1</smiles><smiles>[SbH3]</smiles>

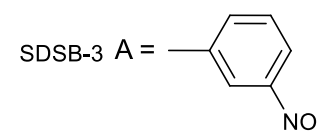<smiles></smiles>

Scheme 2: Syntheses of symmetric double Schiff base

\section{MEASUREMENTS}

Melting points were determined in open capillary tubes and were uncorrected. Purity of the compounds was checked by TLC in appropriate solvent systems. IR spectra ( $\mathrm{KBr}$ pellets) were scanned on a Shimadzu FTIR- 8400 spectrophotometer. The ${ }^{1}$ HNMR spectra of Schiff bases were scanned on a Brucker FTNMR (300 MHz) spectrometer by using $\mathrm{CDCl}_{3}$ as a solvent and TMS as an internal standard. Mass spectra of the compounds were scanned on a Shimadzu GC-MS-QP 2010 spectrometer by using E.I. $(0.7 \mathrm{kV})$ detector. The ion source temperature was $220^{\circ} \mathrm{C}$ and interface temperature was $240^{\circ} \mathrm{C}$. UV spectra were scanned on a Shimadzu UV-1700 spectrophotometer by using $\mathrm{CHCl}_{3}$ and DMSO as solvents. Microbial activity of the compounds was checked by cupplate technique [30].

\section{RESULTS AND DISCUSSION:}

From Table-1 it is observed that reaction time for conventional method is pretty long (7-8h) while it is much smaller for microwave irradiation method (7-8min). UV absorption of SDSB-1 to SDSB-4 was studied in chloroform and dimethyl formamide solvents at room temperature. Schiff bases showed longer wave length of absorption in DMSO system as compared to chloroform system. Thus, DMSO solution displayed blue shift and solvent polarity affect UV absorption. IR, ${ }^{1} \mathrm{HNMR}$ and MS data of SDSB-1 to SDSB-4 are as follow. 
Table 1: Experimental detail for the synthesis of SDSB-1 to SDSB-4

\begin{tabular}{|c|c|c|c|c|c|}
\hline \multirow{2}{*}{ Schiff base } & \multicolumn{2}{|c|}{ Conventional method } & \multicolumn{2}{|c|}{ Microwave irradiation method } & $\begin{array}{c}\text { Crystallization } \\
\text { solvent system }\end{array}$ \\
\cline { 2 - 5 } & Time, h & \% Yield & Time, min & \% Yield & Chloroform -n Hexane \\
\hline SDSB-1 & 7.5 & 68 & 8 & 90 & Ethyl acetate -n-Hexane \\
\hline SDSB-2 & 8.0 & 72 & 7 & 95 & Ethyl acetate -n-Hexane \\
\hline SDSB-3 & 8.0 & 74 & 7 & 95 & Chloroform $-\mathrm{n}$ Hexane \\
\hline SDSB-4 & 7.0 & 70 & 8 & 94 & \\
\hline
\end{tabular}

Table 2: Physical and analytical data of SDSB-1 to SDSB-4

\begin{tabular}{|c|l|c|c|l|c|}
\hline Schiff bases & \multicolumn{1}{|c|}{ M.F. } & M.W. & m. p., ${ }^{\mathbf{0}} \mathbf{C}$ & \multicolumn{1}{|c|}{ Color } & R falue \\
\hline SDSB-1 & $\mathrm{C}_{36} \mathrm{H}_{34} \mathrm{~N}_{2}$ & 494 & 232 & Light brown & c -0.59 \\
\hline SDSB-2 & $\mathrm{C}_{32} \mathrm{H}_{28} \mathrm{~N}_{4} \mathrm{O}_{4}$ & 532 & 258 & Yellow & a-0.68 \\
\hline SDSB-3 & $\mathrm{C}_{32} \mathrm{H}_{28} \mathrm{~N}_{4} \mathrm{O}_{4}$ & 532 & 260 & Yellow & a- 0.71 \\
\hline SDSB-4 & $\mathrm{C}_{36} \mathrm{H}_{40} \mathrm{~N}_{4}$ & 528 & 248 & Light brown & b-0.56 \\
\hline
\end{tabular}

a: Ethyl acetate $-\mathrm{n}$-Hexane $(65: 35 \mathrm{~V} / \mathrm{V})$ b: Ethyl acetate $-\mathrm{n}$-Hexane $(80: 20 \mathrm{~V} / \mathrm{V})$

c: Ethyl acetate $-\mathrm{n}-\mathrm{Hexane}(75: 25 \mathrm{~V} / \mathrm{V})$

\subsection{Characterizations of the Schiff bases}

\section{SDSB-1:}

UV $\left(\lambda_{\text {max }}, \mathbf{n m}\right): \mathrm{CHCl}_{3}$ 255.4, DMSO 263.4

IR $\left(\mathrm{cm}^{-1}\right): \mathrm{CH}=\mathrm{N} 1626.8, v_{\mathrm{C}-\mathrm{N}} 1362.6, v_{\mathrm{C}=\mathrm{C}} 1609.6,1577.7, v_{-\mathrm{CH} 2} \quad 2929.7, \quad 2854.5, v_{\mathrm{C}-\mathrm{H}}(-\mathrm{oopd})$ $814.9 v_{\mathrm{C}-\mathrm{H}}(-\mathrm{ipd}) 1106.1,1075.2$

MS: 490

\section{SDSB-2:}

UV $\left(\boldsymbol{\lambda}_{\text {max }}, \mathbf{n m}\right): \mathrm{CHCl}_{3}$ 246.0, DMSO 261.4

IR $\left(\mathrm{cm}^{-1}\right): \quad \mathrm{CH}=\mathrm{N} 1614.3, v_{\mathrm{C}-\mathrm{N}} 1305.7, v_{\mathrm{C}=\mathrm{C}} 1568.0,1519.8, v_{-\mathrm{CH} 2} 2931.6,2856.4, v_{\mathrm{C}-\mathrm{H}}$ (-oopd) 829.3, $v_{\text {C-H }}($-ipd) $1174.6,1109.0$

${ }^{1}$ H NMR (ppm): $1.611\left(6 \mathrm{H}, \mathrm{s}, \beta+\gamma,-\mathrm{CH}_{2}-\right), 2.329\left(4 \mathrm{H}, \mathrm{s}, \alpha,-\mathrm{CH}_{2}-\right), 7.294-7.286\left(4 \mathrm{H}, \mathrm{d}, \mathrm{Ar}-\mathrm{H}_{\mathrm{b}}\right.$, $\mathrm{J}=8.5$ )

7.244 $\left(\mathrm{CDCl}_{3}\right), 7.373-7.344\left(2 \mathrm{H}, \mathrm{d}, \mathrm{Ar}-\mathrm{H}_{\mathrm{a}} \mathrm{J}=8.5\right), 7.617-7.561\left(2 \mathrm{H}, \mathrm{m}, \mathrm{Ar}-\mathrm{H}_{\mathrm{e}}, \mathrm{J}=8.4\right), 7.738-$ $7.688\left(2 \mathrm{H}, \mathrm{m}, \mathrm{Ar}-\mathrm{H}_{\mathrm{f}}, \mathrm{J}=7.5\right), 8.066-8.035\left(2 \mathrm{H}, \mathrm{d}, \mathrm{Ar}-\mathrm{H}_{\mathrm{g}}, \mathrm{J}=9.4\right), 8.298-8.270\left(2 \mathrm{H}, \mathrm{d}, \mathrm{Ar}-\mathrm{H}_{\mathrm{d}}\right.$, $\mathrm{J}=8.5), 8.942(2 \mathrm{H}, \mathrm{s},-\mathrm{CH}=\mathrm{N}-)$

MS: 532 
SDSB-3:

UV $\left(\lambda_{\text {max }}, \mathbf{n m}\right): \mathrm{CHCl}_{3}$ 245.2, DMSO 266.4

IR $\left(\mathrm{cm}^{-1}\right): \mathrm{CH}=\mathrm{N} 1626.8, v_{\mathrm{C}-\mathrm{N}} 1352.0, v_{\mathrm{C}=\mathrm{C}} \quad 1573.8,1498.6 v_{-\mathrm{CH} 2} 2914.2,2843.8, v_{\mathrm{C}-\mathrm{H}}$ (-oopd) 813.9. $v_{\text {C-H }}($-ipd) $1171.7,1074.3$

${ }^{1}$ H NMR (ppm): 1.599 (6H, s, $\left.\beta+\gamma,-\mathrm{CH}_{2}-\right), 2.329$ (4H, s, $\left.\alpha,-\mathrm{CH}_{2}-\right), 7.211-7.183$ (4H,d, Ar- $\mathrm{H}_{\mathrm{b}}$, $\mathrm{J}=8.4)$

$7.292\left(\mathrm{CDCl}_{3}\right), 7.372-7.343\left(4 \mathrm{H}, \mathrm{d}, \mathrm{Ar}-\mathrm{H}_{\mathrm{a}} \mathrm{J}=8.6\right), 7.647-7.597\left(2 \mathrm{H}, \mathrm{t}, \mathrm{Ar}-\mathrm{H}_{\mathrm{f}}, \mathrm{J}=7.4,7.7\right), 8.224-$ $8.196\left(2 \mathrm{H}, \mathrm{d}, \mathrm{Ar}-\mathrm{H}_{\mathrm{g}}, \mathrm{J}=8.3\right) 8.296-8.267\left(2 \mathrm{H}, \mathrm{d}, \mathrm{Ar}-\mathrm{H}_{\mathrm{e}}, \mathrm{J}=7.9\right), 8.529\left(2 \mathrm{H}, \mathrm{s}, \mathrm{Ar}-\mathrm{H}_{\mathrm{d}},\right), 8.705(2 \mathrm{H}$, $\mathrm{s},-\mathrm{CH}=\mathrm{N}-)$

MS: 532

\section{SDSB-4:}

UV ( $\left.\boldsymbol{\lambda}_{\text {max }}, \mathbf{n m}\right): \mathrm{CHCl}_{3}$ 361.4, DMSO 363.4

IR $\left(\mathrm{cm}^{-1}\right): \mathrm{CH}=\mathrm{N} 1606.6, v_{\mathrm{C}-\mathrm{N}} 1312.5, v_{\mathrm{C}=\mathrm{C}} 1606.6, v_{-\mathrm{CH} 2} 2930.6,2853.5, v_{\mathrm{C}-\mathrm{H}}(-\mathrm{oopd}) 814.9$, $v_{\mathrm{C}-\mathrm{H}}(-\mathrm{ipd}) 1165.9,1123.5$

${ }^{1}$ H NMR (ppm): $1.575\left(6 \mathrm{H}, \mathrm{s}, \beta+\gamma,-\mathrm{CH}_{2^{-}}\right), 2.291\left(4 \mathrm{H}, \mathrm{s}, \alpha,-\mathrm{CH}_{2^{-}}\right), 3.019\left(12 \mathrm{H}, \mathrm{s}, \mathrm{H}_{\mathrm{f}}\right), 6.722-$ $6.693\left(4 \mathrm{H}, \mathrm{d}, \mathrm{Ar}-\mathrm{H}_{\mathrm{b}} \mathrm{J}=8.7\right), 7.137-7.109\left(4 \mathrm{H}, \mathrm{d}, \mathrm{Ar}-\mathrm{H}_{\mathrm{e}}, \mathrm{J}=8.5\right), 7.238\left(\mathrm{CDCl}_{3}\right), 7.291-7.263(4 \mathrm{H}$, $\left.\mathrm{d}, \mathrm{Ar}-\mathrm{H}_{\mathrm{d}} \mathrm{J}=8.4\right)$,

7.766-7.737 (4H, d, Ar- $\left.\mathrm{H}_{\mathrm{a}}, \mathrm{J}=8.7\right), 8.314(2 \mathrm{H}, \mathrm{s},-\mathrm{CH}=\mathrm{N}-)$

MS: 528

Pyrolysis of compounds is a complex process and involves a variety of reactions such as ionization, decomposition, branching, cross-linking and rearrangement. Mass spectral fragmentation of the Schiff base is shown in Scheme-3. Important fragments are presented Tables-3. Loss of electron results in to molecular ion, while loss of azomethine proton gives $(\mathrm{M}-1)^{+}$ion peak in the mass spectrum. (M-1) $)^{+}$ion undergoes further fragmentation to give fragments 1 and 2 . Fragment 1 further undergoes fragmentation to give fragments 3 and 4. Similarly fragment - 2 produces fragments - 4 and 6, while fragment - 3 produces fragments 5, 6 and 7. In case of main fragments, many more fragments are possible due to above mentioned possible reactions. At the end all fragments are converted into low molecular mass gaseous products. 

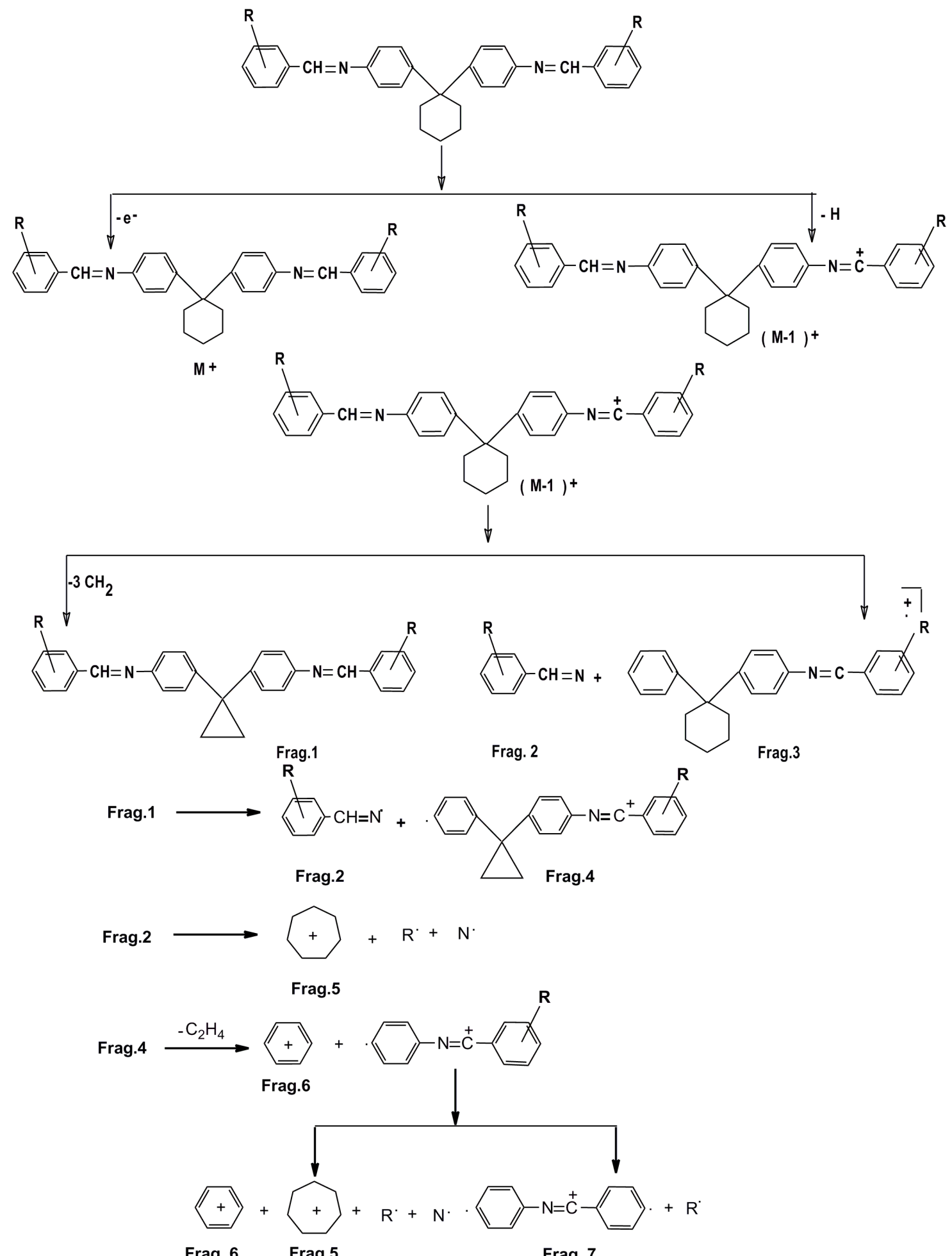

Frag. 6 Frag.5

Frag. 7 
Table 3: Molecular ion $\left(\mathrm{M}^{+}\right),(\mathrm{M}-1)^{+}$peaks and some important Fragments of SDSB-1 to SDSB-4.

\begin{tabular}{|c|c|c|c|c|c|c|c|c|c|}
\hline Schiff base & $\mathbf{M}^{+}$ & (M-1) $^{+}$ & F-1 & F-2 & F-3 & F-4 & F-5 & F-6 & F-7 \\
\hline SDSB -1 & 490 & - & 448 & 130 & 360 & 318 & 91 & 77 & 178 \\
\hline SDSB -2 & 532 & 531 & 490 & 149 & 383 & 341 & 91 & 77 & 178 \\
\hline SDSB-3 & 532 & 531 & 490 & 149 & 383 & 341 & 91 & 77 & 178 \\
\hline SDSB -4 & 528 & 527 & 486 & 147 & 381 & 339 & 91 & 77 & 178 \\
\hline
\end{tabular}

\section{ANTIMICROBIAL ACTIVITY}

Schiff bases, standard drugs (amoxicillin, ampicillin, ciprofloxacin and erythromycin) and solvent DMF were screened for their microbial activity against Escherichia coli (E.coli), Bacillus megaterium (B. mega), Proteus Vulgeris (P.vulgeris), Staphylococcus aureus (S.aureus) and Aspergillus niger (A. niger) by cup plate method at $37^{\circ} \mathrm{C}$. The sample volume was $0.1 \mathrm{ml}(50 \mu \mathrm{g})$. The plates were incubated for $24 \mathrm{~h}$ at $37^{\circ} \mathrm{C}$ and zones of inhibition for each microbial growth were measured relative to control (DMF) and are reported in Table 4, from which it is observed that SDSB-1 to SDSB-4 showed moderate antibacterial activity in comparison with standard drugs. SDSB-1 showed moderate antifungal activity against A. niger and erythromycin but SDSB-2 to SDSB-4 showed comparable activity. The significant antifungal activity is due to presence of nitro groups and $\mathrm{N}, \mathrm{N}$-dimethyl groups in the Schiff bases.

Table 4: A comparative zone of inhibition for standard drugs and Schiff bases

\begin{tabular}{|c|c|c|c|c|c|}
\hline \multirow{2}{*}{ Sample } & \multicolumn{5}{|c|}{ Zone of inhibition, mm } \\
\cline { 2 - 6 } & $\begin{array}{c}\text { E. coli } \\
\text { MTCC-443, }\end{array}$ & $\begin{array}{c}\text { B. mega } \\
\text { MTCC-244 }\end{array}$ & $\begin{array}{c}\text { B. sub. } \\
\text { MTCC-441 }\end{array}$ & $\begin{array}{c}\text { P. vulgaris } \\
\text { MTCC-744 }\end{array}$ & $\begin{array}{c}\text { A. niger } \\
\text { MTCC-282 }\end{array}$ \\
\hline SDSB -1 & 7 & 9 & 8 & 9 & 12 \\
\hline SDSB -2 & 9 & 9 & 11 & 10 & 15 \\
\hline SDSB -3 & 9 & 9 & 10 & 11 & 16 \\
\hline SDSB -4 & 8 & 10 & 9 & 10 & 16 \\
\hline Amopicillin & 18 & 19 & 22 & 22 & - \\
\hline Amoxicillin & 17 & 21 & 19 & 23 & - \\
\hline Ciprofloxacin & 22 & 20 & 21 & 24 & - \\
\hline Erythromycin & 19 & 20 & 22 & 24 & 18 \\
\hline
\end{tabular}

\section{CONCLUSIONS}

As compared to conventional method microwave irradiated method led to shorter reaction time and improved reaction yields. Schiff bases showed moderate antibacterial activity and moderate to comparable antifungal activity against chosen microbes and standard drugs.

\section{Acknowledgements}

The auther are thankful to the Director SAIF, Punjab University, Chandigarh for NMR analysis and the Director CDRI, Lukhnow for FAB mass spectral analysis. 


\section{References}

[1] S. Patai The chemistry of the carbon-nitrogen double bond. (John-Wiley and Sons. Ltd. London (1970)

[2] R.W. Layer, Chem Rev., 63 (1963) 487-507.

[3] A. P. Mishra, M. Khare, S. K. Gautam, Synth. React Inorg. Met-Org Chem., 32(2002)14851500 .

[4] C. Bi, Y. Fan, Synth. React Inorg and Met-Org Chem. 34 (2004) 687-695.

[5] M. G. Bhowan, T. Jeewoth and H. L. Kam-wah, Transition Metal Chem., 24 (1999) 445-448.

[6] S. H. Abdel-Hafez, Phosphorus, sulfur, and silicon. 178(2003) 2563-2579.

[7] V. S. Agarwala. A.R. Krishnaswamy, P. K. Sen, U.S. pat.5, 147, 567(1992)

[8] M. D. Tarafder, A. Kasbollah, N. Saravanan, K. A. Crouse, A. M. Ali, K. T. Oo, J. Biochem., Mol. bio. and Biophys., 6 (2002)85-91.

[9] K. D. Patel, B. D. Mistry, K. R. Desai, J. Ind. Chem. Soc., 81(2004) 783-785.

[10] Yu. V. Popov, T. K. Korchagina, G. V. Chicherina, Russian J. Org. Chem., 37 (2001) 677679.

[11] V. E. Kuzmin, V. P. Lozitsky, G. L. Kamalov, R. N. Lozitskaya, A. I. Zheltvay, A. S. Fedtchouk, D. N. Kryzhanovsky, Acta Biochimica, Polonica, 47(2000)867-875.

[12] V. P. Lozitsky, V. E. Kuzmin, A. G. Artemenko, R. N. Lozytska, A. S. Fedtchouk., E. N. Muratov, A. K. Mescheriakov, SAR and QSAR in Environmental Research, 16(2005) 219230.

[13] H. Temel, J. Coord. Chem., 5(2004) 723-729.

[14] R. S. Varma. Green Chem., 1 (1999)43-55.

[15] M. M. Aghayan, Synth. Commun.,33(2003) 521-525.

[16] A. Loupy, A. Petit, I. Hamelin, F. Texier-Boullet, P. Jacquault, D. Mathe, Synthesis (1998) 1213-1234.

[17] M. Lamiri , K. Bougrin, B. Daou, M. Souflaoui, E. Nicolas, E. Giralt, Synth. Commun. 36 (2006)1575-1584.

[18] Y. X. Li , W. G. Zhao,Z. M. Li , S. H. Wang, W. L. Dong, Synth. Commun, 36(2006) 1471-1477.

[19] M. Xia, S.H. Wang, W. Yuan, Synth. Commun., 34, (2004)3175-3182.

[20] R. Gedye, F. Smith, K. Westaway, H. Ali, L. Laberege, L. Baldisera, J. Roussel, Tetrahedron Lett. 27(1986) 279-282.

[21] S. Caddic, Tetrahedron., 53(1995)10403-10432.

[22] H. Yang, W. H. Sun, Z. Li, L. Wang, Synth. Commun., 32 (2002)2395-2402.

[23] S. K. Hamilton, D.E. Wikinson, G. S. Hamiton,Y. Q. Wu, Org. Lett., 7 (2005)2429-2431.

[24] M. J. Lin, C.M. Sun, Tetrahedron Lett. 44(2003) 8739-8742.

[25] K.Tanka, R. Shirashi, Green Chem. 2(2000) 272-273

[26] J. Zheng, X Guo, Z. Yang, Synth. Comm., 34(2004)4517-4520.

[27] M. H. Yi, W. Hung, M. Y. Jin, K. Y. Choi, Macromolecules, 30(1997)5606-5611.

[28] B. J. Gangani, P. H. Parsania, Spectroscopy Letters, 40(2007) 97-112.

[29] B. J. Gangani, P. H. Parsania, International Letters of Chemistry, Physics and Astronomy, 57, (2015) 36-44,

[30] A. L. Bary, The antimicrobial susceptibility test, principle and practice, 180 193(Philadelphia, 1976) 